\title{
Editorial
}

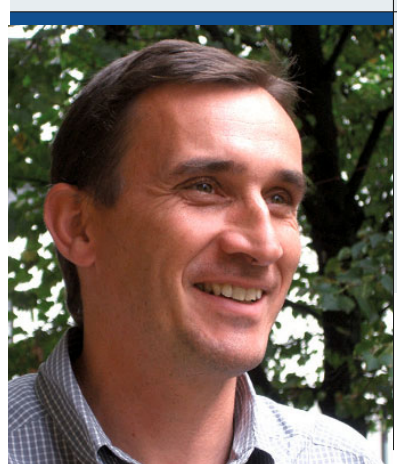

„Die Andrologie ist uns - neben Dermatologen und

Endokrinologen - als Zusatzbezeichnung gesichert"

Ulrich Pickl, niedergelassener Urologe mit der

Zusatzbezeichnung: Andrologie, Psychotherapie und

Sexualmedizin (Akademie für Sexualmedizin) in München

\section{Ein breites Tätigkeitsfeld für Urologen: Die Andrologie}

V iele Bereiche der Urologie sind in den letzten Jahren weggebrochen. So wurde die Kinderurologie in vielen Regionen von Kinderärzten/Kinderchirurgen übernommen, die Transplantationschirurgie ging zu den Chirurgen. Die Indikationsstellung zur urologischen Radiologie hat sich verringert, die medikamentöse Tumortherapie ist gerade im Begriff abzuwandern. Wird auch das Labor geringer vergütet, so werden sich viele Urologen neue Betätigungsfelder suchen müssen.

Das Teilgebiet „Andrologie“ ist umfangreich. Es umfasst die konservative und operative Andrologie, Beratung zu assistierter Befruchtung, Störungen des alternden Mannes, ddie schwierige Indikation einer adäquaten Hormonsubstitution bis hin zur Behandlung des metabolischen Syndrom bei Übergewicht. Oft müssen hier psychosoziale Faktoren thematisiert werden, weil sich gesellschaftliche und epidemiologische Strukturen verändert haben. Diese unterschiedlichen Facetten der Andrologie, einschließlich der Sexualmedizin, erhöhen die Attraktivität eines andrologisch tätigen Urologen in Klinik und Praxis. Aber wir Urologen müssen diese Zusatzbezeichnung auch pflegen. Andrologisch tätige Urologen müssen sich als Männerärzte verstehen und definieren, sich als der kompetente Ansprechpartner für den Mann etablieren, wie das die Gynäkologen bei Frauen schon seit langen getan haben. Es darf nicht passieren, dass die Bezeichnung „Männerarzt“ - in Wochenendkursen von Hausärzten, Internisten und Urologen(!) erworben - der Zusatzbezeichnung Andrologie gleichgestellt wird.

Die Fertilität des Mannes wird nicht immer adäquat abgeklärt. Die Andrologen stehen in Konkurrenz zu vielen IVF-Zentren. Dort werden Spermiogramme durchgeführt, zum Teil mit besserer Qualität als bei manchem Urologen. Aber dort findet meist keine körperliche Untersuchung, keine Sexualanamnese statt. Es fehlt die Sonographie des Hodens und Ursachenforschung der männlichen Subfertilität. Die assistierte Befruchtung steht dort im Vordergrund.
Wir sollten die Kooperation mit IVF-Zentren suchen. Dies hat sich vielerorts bewährt bei der Beratung und Abkärung subfertiler Männer. Liegt z.B. eine Azoospermie vor, was bei ca.10-20\% der Männer mit Fertilitätsstörurung vorkommt, so hat sich die offene Hodenbiopsie als überlegen erwiesen gegenüber der meist von Gynäkologen durchgeführten Technik der blinden perkutanen Hodenbiopsie.

Zur Andrologie gehört auch das weite Feld der Sexualmedizin. Sexuelle Funktionsstörungen sind auf Grund ihrer hohen Prävalenz und Inzidenz wichtige Gesundheitsprobleme. Neben der organischen Abklärung sollten auch psychische und psychosomatische Störfaktoren aktiv angesprochen werden. Das Erlernen einer standardisierten, themenzentrierten Gesprächsführung ist unabdingbar in der Sexualmedizin und verringert den Zeitaufwand. Durch eigene Weiterbildung in der Gesprächsführung und Sexualberatung kann der andrologisch tätige Urologe die meisten Patienten mit Sexualstörungen erfolgreich behandeln, auch unter Einbeziehung der Pharmakotherapie. So limitieren wir uns nicht nur als Rezeptierer vasoaktiver Substanzen, sondern qualifizieren uns als kompetente Gesprächspartner.

Erkennt der Patient, dass er mit seinem Andrologen einen gut ausgebildeten Mediziner hat, dann sehe ich auch die Honorierung über IGeL bei einigen Leistungen als gerechtfertigt, nicht aber bei „Männerärzten“, die mit exotischen Laborkonstrukten „AntiAging-Medizin" verkaufen.

Die Andrologie bietet ein breites Betätigungsfeld, welches wir Urologen nur als qualifizierte Männerärzte sichern können. Dies bedingt aber regelmäßige Fortbildung und Teilnahme an Qualitätszirkeln und Ringversuchen.

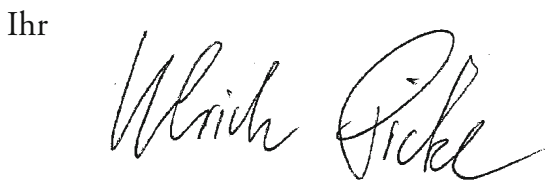

